\title{
openheart Surgical versus balloon valvotomy in neonates and infants: results from the UK National Audit
}

\author{
Dan Mihai Dorobantu, ${ }^{1,2}$ Demetris Taliotis, ${ }^{1}$ Robert Michael Tulloh, ${ }^{1,3}$ \\ Mansour Thagavi Azar Sharabiani, ${ }^{4}$ Eltayeb Mohamed Ahmed, ${ }^{3}$ \\ Gianni Davide Angelini, ${ }^{1,3}$ Serban Constantin Stoica ${ }^{1,3}$
}

\begin{abstract}
To cite: Dorobantu DM, Taliotis D, Tulloh RM, et al. Surgical versus balloon valvotomy in neonates and infants: results from the UK National Audit. Open Heart 2019;6:e000938. doi:10.1136/ openhrt-2018-000938
\end{abstract}

Received 28 September 2018 Revised 6 December 2018 Accepted 9 December 2018

(A) Check for updates

(c) Author(s) (or their employer(s)) 2019. Re-use permitted under CC BY-NC. No commercial re-use. See rights and permissions. Published by BMJ.

${ }^{1}$ Departments of Cardiac Surgery and Cardiology, Bristol Royal Hospital for Children, Bristol, UK

${ }^{2}$ Cardiology Department, "Prof. C.C. Iliescu" Emergency Institute for Cardiovascular Diseases, Bucharest, Romania ${ }^{3}$ Departments of Cardiac Surgery and Cardiology, Bristol Heart Institute, Bristol, UK ${ }^{4}$ School of Public Health, Imperial College, London, UK

Correspondence to Dr Dan Mihai Dorobantu; dn. dorobantu@gmail.com

\section{ABSTRACT}

Objective There are conflicting data on choosing balloon aortic valvoplasty (BAV) or surgical aortic valvotomy (SAV) in neonates and infants requiring intervention for aortic valve stenosis. We aim to report the outcome of both techniques based on results from the UK national registry. Methods This is a retrospective study, including all patients under 1 year undergoing BAV/SAV between 2000 and 2012. A modulated renewal approach was used to examine the effect of reinterventions on outcomes. Results A total of 647 patients (488 BAV, 159 SAV, 292 neonates) undergoing 888 aortic valve procedures were included, with a median age of 40 days. Unadjusted survival at 10 years was $90.6 \%$ after initial BAV and $84.9 \%$ after initial SAV. Unadjusted aortic valve replacement (AVR) rate at 10 years was $78 \%$ after initial BAV and $80.3 \%$ after initial SAV. Initial BAV and SAV had comparable outcomes at 10 years when adjusted by covariates $(p>0.4)$. AVR rates were higher after BAV and SAV reinterventions compared with initial valvoplasty without reinterventions (reference BAV, $\mathrm{HR}=3$ and 3.8 , respectively, $\mathrm{p}<0.001$ ). Neonates accounted for $29 / 35$ of early deaths after the initial procedure, without significant differences between BAV and SAV, with all late outcomes being worse compared with infants $(p<0.005)$.

Conclusions In a group of consecutive neonates and infants, BAV and SAV had comparable survival and freedom from reintervention as initial procedures and when performed as reinterventions. These findings support a treatment choice based on patient characteristics and centre expertise, and further research into the best patient profile for each choice.

\section{INTRODUCTION}

Congenital aortic valve stenosis (AS) is among the more common congenital heart defects, accounting for $6 \%$ of all congenital heart disease. ${ }^{1}$ When the stenosis is severe, it requires intervention during the neonatal life or early infancy. This is undertaken either with a balloon aortic valvoplasty (BAV) or surgical aortic valvotomy (SAV). Throughout the last decades clinicians have switched from one preferred option to another, as both techniques evolved. ${ }^{2}$ BAV offers the advantage of

\section{Key questions}

What is already known about this subject?

- Balloon aortic valvoplasty (BAV) and surgical aortic valvotomy (SAV) are used with good results in neonates and infants, but there is ongoing debate on whether one method achieves better outcomes than the other, with clinical practices varying considerably.

What does this study add?

- The current study compares BAV and SAV in a national population of 647 neonates and infants, bringing patients from various practices, spanning over a decade.

- BAV and SAV were found to have comparable survival and freedom from reintervention at 10 years. Unadjusted survival at 10 years was $90.6 \%$ after initial BAV and $84.9 \%$ after initial SAV.

- Unadjusted aortic valve replacement rate at 10 years was $78 \%$ after initial BAV and $80.3 \%$ after initial SAV. Neonatal procedures associated cardiac defects and reinterventions are associated with worse outcomes.

How might this impact on clinical practice?

- The choice between BAV and SAV is currently taken in an individualised manner, in a multidisciplinary meeting.

- The fact that we found similar results with both methods supports this practice. Nevertheless, we identified subgroups of patients, namely neonates and those with associated cardiac defects, where outcomes are worse.

- Future research should be focused on determining clinical and imaging criteria to aid treatment choice.

avoiding a sternotomy and performing the procedure without the need for cardiopulmonary bypass in a child with an impaired ventricular function, where the surgical risk might be prohibitive. SAV permits for direct visualisation of the valve and repair of the valve, if possible.

Currently, most centres in the UK have experience with both strategies, but tend to 
favour the one with the best institutional results. This is mirrored in the available international published data, summarised in a recent meta-analysis, with only four single-centre and two multicentre comparative reports, compared with 12 reports of a single technique, as

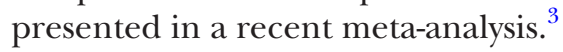

In a landmark study published by the Congenital Heart Surgeons Society in 2001, BAV and SAV were shown to have similar outcomes in neonates, but whether the same is true in present times or in infant patients is debatable. ${ }^{4}$ The publishing of this study was followed by an increased interest in BAV. A decade later several single-centre series showed superior results with the surgical approach, ${ }^{5-7}$ and the use of SAV has regained momentum in a true 'pendulum swing'. ${ }^{2}$ These variations in preferences and use make applying current data difficult in day-to-day practice. In addition, few studies, if any, include large enough patient groups undergoing reintervention or with associated cardiac defects to allow for a meaningful analysis of more complex management pathways.

A multicentre registry, such as the UK National Congenital Heart Disease Audit (NCHDA) is well equipped to show the contemporary picture of aortic valve intervention, by including centres with diverse practices, while gathering sufficient numbers to allow for adjustments for patient characteristics. Our study aims to: (1) report national early and late outcomes after initial BAV or SAV and aortic valve reintervention; 2) identify predictors for late mortality and reintervention; (3) describe the outcomes in the neonatal and associated cardiac defects subgroups.

\section{PATIENTS AND METHODS \\ The dataset}

The National Institute for Cardiovascular Outcomes Research (NICOR) collects validated key data on cardiac procedures from all the UK units in the NCHDA (https: / / nicor4.nicor.org.uk/). Linkage with survival registries of Northern Ireland and Scotland cannot be performed consistently, while a minority of patients are private patients from abroad. This resulted in 7.7\% (n=50) of patients not having follow-up data beyond 30 days. The remaining patients have follow-up from the Office of National Statistics and/or subsequent entries in the database. The need for patient-level consent to participate in this retrospective study with anonymised data was waived by the NICOR Research Board.

\section{Patient selection and classification}

All available data in the registry on 727 patients either an initial BAV or a SAV at under 1 year of age between 1 April 2000 and 31 March 2012 were selected and anonymised. We excluded 53 patients directed towards a single ventricle pathway and 27 patients previous procedures, resulting in a final group of 647 infants and 888 aortic valve procedures.
Reintervention was defined as any aortic valve procedure, surgical or transcatheter, following an index (first) $\mathrm{BAV} / \mathrm{SAV}$ procedure. Reinterventions were divided into BAV, SAV or aortic valve replacement (AVR) subgroups. Early events are defined as occurring within 30 days from the index procedure.

\section{Statistical analysis}

Frequencies are given as numbers and percentages, continuous values as median (IQR). Population characteristics were compared using the Mann-Whitney U test, Kruskal Wallis test and the Fisher's exact test. As most variables were not normally distributed (tested with the Shapiro-Wilks test), we used non-parametrical tests throughout. Unadjusted survival and freedom from reintervention at 10 years are estimated using the KaplanMeier method, considering the time from the procedure of interest (initial BAV/SAV or reintervention) up to the first event of each type (death, AVR, aortic valvoplasty or valvotomy) and presented as percentage and 95\% CI.

Patients with an associated cardiac defect had a higher likelihood of being selected for a surgical approach, regardless of other factors. To assess this possible source of bias, we analysed outcomes in two subgroups: isolated aortic valve disease $(\mathrm{n}=454,70 \%)$ and aortic valve disease with an associated defect $(n=193,30 \%)$. The latter group of patients were matched by age, weight-for-age $\mathrm{z}$ score, gender and associated cardiac defects using a coarsened exact matching algorithm ('cem' package for STATA) and analysis repeated. ${ }^{8}$

To evaluate the effect of the initial procedure and the effect of reinterventions on outcomes, we used a modulated renewal approach when building the models, resetting the time-to-event variable after each reintervention and creating a new entry and retaining a number of timevarying variables: type of current procedure or reintervention, age at the current procedure and weight-for-age z score at current procedure. ${ }^{9}$

We used multivariable survival and freedom from reintervention Weibull regression models in the whole study population, using a stepwise backwards elimination method, including the following variables: current procedure type (categorical with five values-initial BAV/SAV, $\mathrm{BAV} / \mathrm{SAV}$ reintervention, AVR), initial age (continuous), neonate at initial procedure, initial weight-for-age z score, current procedure age, current procedure weight-for-age $\mathrm{z}$ score, patient gender, coarctation of the aorta $(\mathrm{CoA})$, mitral valve abnormality, ventricular septal defect, atrial septal defect, subaortic stenosis, right ventricular outflow tract obstruction, genetic syndrome. At each step, the variable with the higher $\mathrm{p}$ value was excluded, until all remaining ones had a $\mathrm{p}<0.05$, in order to avoid spurious results. Additionally, we performed subgroup analyses of outcomes in neonates and patients with associated cardiac defects. Neonates and patients with associated defects were identified as potential bias sources, and as such sensitivity analysis was performed by excluding 


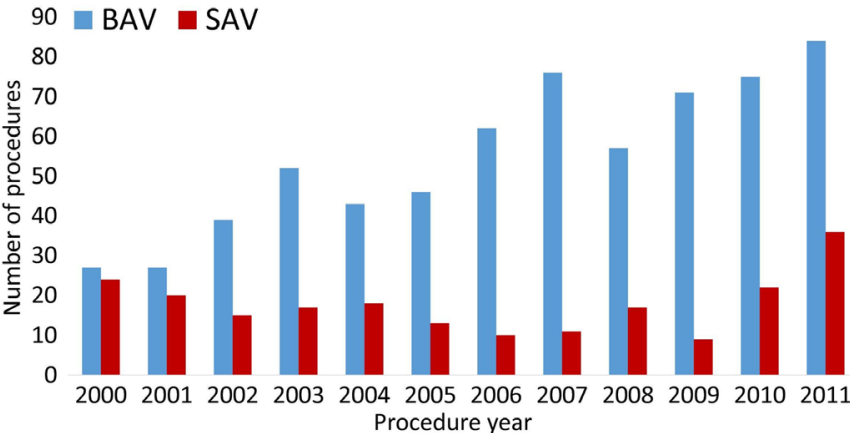

Figure 1 Initial aortic valve procedure (BAV or SAV) trends between 2000 and 2011 in the UK. There was a steady increase of BAV performed, while the number of SAV decreased up to the middle of the last decade and rose in the latter half. BAV, balloon aortic valvoplasty; SAV, surgical aortic valvotomy.

each subgroup and repeating the late outcomes analysis, without significant changes to results (data not shown).

Statistical analyses were done with STATA/IC 11.2 (Stata, College Station, Texas, USA). Patient weightfor-age z-score was calculated using the WHO macro for STATA (http://www.who.int/childgrowth/software/ en/).

\section{RESULTS}

A total of 647 neonates and infants (488 BAV, 159 SAV) undergoing 888 aortic valve procedures were included. During the first half of the last decade, usage of SAV in the UK has decreased in this population, but in the second half there has been an increase in the number of children undergoing SAV, translating in an increasing proportion relative to BAV (figure 1). Additionally, figure 1 shows a steady increase in the number of patients treated each year. Table 1 shows patient and initial procedure characteristics. The observed treatment pathways and time to reinterventions are summarised in figure 2, with a total of 98 reintervention BAV, 59 reintervention SAV and 84 AVR.

Few patients $(<2 \%)$ have more than two reinterventions during the observed follow-up. Median age at BAV reintervention is 5.7 months $(3.2 ; 19.9)$, at reintervention SAV 7.7 months $(1.3 ; 19)$ and at AVR 15 months $(3.1 ; 41.2)$. Significantly more SAV reinterventions are performed at less than 30 days from a previous AoV procedure $(n=20$, $33.9 \%$ vs $\mathrm{n}=14,14.3 \%$ for $\mathrm{BAV}$ and $\mathrm{n}=13,15.5 \%$ for $\mathrm{AVR}$, $\mathrm{p}<0.001)$.

Among the 84 AVR procedures recorded, there were 75 (89.3\%) Ross/Ross-Konno operations, 3 (3.6\%) homograft AVRs, 2 mechanical AVRs (2.4\%), 1 bioprosthesis AVR (1.2\%) and 3 unknown AVRs (3.6\%).

\section{Early outcomes}

No significant differences were found between initial or reintervention BAV and SAV choice in terms of early outcomes. Mortality at 30 days after the initial procedure was $5.9 \%$ overall: $5.7 \%$ after initial BAV, $6.5 \%$ after initial

\begin{tabular}{|c|c|c|c|}
\hline Initial procedure & BAV $(n=488)$ & SAV $(n=159)$ & $P$ value \\
\hline $\begin{array}{l}\text { Age, days (median, } \\
\text { IQR) }\end{array}$ & $45(7.8 ; 101)$ & $27(9 ; 102)$ & 0.48 \\
\hline $\begin{array}{l}\text { Weight-for-age Z score } \\
\text { (median IQR) }\end{array}$ & $-0.7(-1.7 ; 0.2)$ & $-0.9(-2 ; 0.2)$ & 0.61 \\
\hline Male gender $(n, \%)$ & $358(73.4 \%)$ & $112(70.4 \%)$ & 0.47 \\
\hline Associated defects & $113(23.2)$ & $80(50.3)$ & $<0.001$ \\
\hline $\mathrm{CoA}$ & $40(8.2)$ & $36(22.6)$ & $<0.001$ \\
\hline MV abnormality & $37(7.6)$ & $16(10.1)$ & 0.35 \\
\hline SAS & $21(4.3)$ & $14(8.8)$ & 0.04 \\
\hline ASD & $16(3.3)$ & $12(7.6)$ & 0.02 \\
\hline VSD & $12(2.5)$ & $26(16.3)$ & $<0.001$ \\
\hline RVOTO & $10(2.1)$ & $8(5)$ & 0.05 \\
\hline Genetic syndromes & $7(1.4)$ & $5(3.1)$ & 0.18 \\
\hline
\end{tabular}

Concomitant

procedures

\begin{tabular}{|c|c|c|c|}
\hline CoA correction & $10(2.1)$ & $26(16.3)$ & $<0.001$ \\
\hline $\begin{array}{l}\text { MV repair/ } \\
\text { replacement }\end{array}$ & 0 & $3(1.9)$ & 0.01 \\
\hline SAS relief & 0 & $6(3.8)$ & $<0.001$ \\
\hline ASD closure & 0 & $3(1.9)$ & 0.01 \\
\hline VSD closure & 0 & $4(2.5)$ & 0.004 \\
\hline $\begin{array}{l}\text { Follow-up, years } \\
\text { (median, IQR) }\end{array}$ & $4.6(2.1 ; 8.2)$ & $2.9(0.7 ; 8.8)$ & 0.06 \\
\hline
\end{tabular}

ASD, atrial septal defect; CoA, coarctation of the aorta; MV, mitral valve; RVOTO, right ventricular outflow tract obstruction; SAS, subaortic stenosis; VSD, ventricular septal defect.

SAV, 3.2\% after BAV reintervention and $9.4 \%$ after SAV reintervention $(\mathrm{p}=0.5)$. Early AVR risk was $1.7 \%$ overall; $1.5 \%$ after initial BAV, $2.2 \%$ after initial SAV, $1.1 \%$ after BAV reintervention and $1.8 \%$ after SAV reintervention $(\mathrm{p}=0.9)$. Risk for early non-AVR valve reintervention was $5.4 \%$ overall, $5.9 \%$ after initial BAV, $3.6 \%$ after initial SAV, $1.1 \%$ after BAV reintervention and $1.9 \%$ after SAV reintervention $(\mathrm{p}=0.13)$.

\section{Late survival}

The unadjusted survival at 10 years was $89.3 \%$ (86.5; $91.5 \%), 90.6 \%(87.5 ; 92.9 \%)$ after initial BAV and $84.9 \%$ $(77.7 ; 89.9 \%)$ after initial SAV. We found no significant differences between the choice of BAV or SAV as initial procedure or reintervention, but we did find a significantly higher mortality at 10 years after an AVR type reintervention (adjusted $\mathrm{HR}=2.8, \mathrm{p}=0.02$, initial $\mathrm{BAV}$ as reference, figure $3 \mathrm{~A})$.

\section{Freedom from AVR}

The unadjusted freedom from AVR at 10 years was $78.7 \%$ (73.3;83.2\%), $78 \%(71.3 ; 83.3 \%)$ after initial BAV and $80.3 \%(69.2 ; 87.7 \%)$ after initial SAV. BAV and SAV performed as reintervention were associated with increased risk for subsequent AVR (adjusted $\mathrm{HR}=1.8$, 


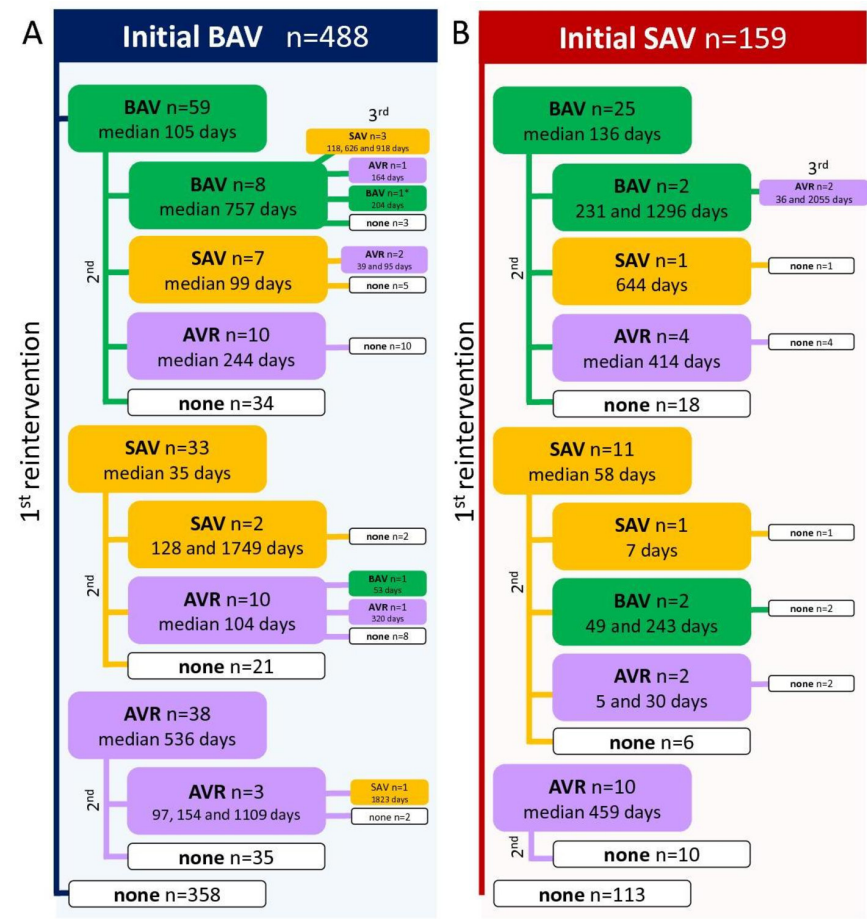

Figure 2 Summary of the aortic valve procedures performed in the initial BAV group (A) and the initial SAV group (B), by order of reinterventions (first on the left, second below and middle and third to the right). Numerical values represent time from the previous procedure in days (median if $>3$ similar reinterventions, each separate value if three or less). Colour coding follows each index procedure (blue for initial BAV, red for initial SAV, green for reintervention BAV, orange for reintervention SAV and light purple for AVR). ${ }^{*}$ There is one patient with a fourth reintervention, an AVR at 202 days following a BAV. AVR, aortic valve replacement; BAV, balloon aortic valvoplasty; SAV, surgical aortic valvotomy.

$\mathrm{p}=0.05$ and $\mathrm{HR}=2.6, \mathrm{p}=0.003$ respectively, initial $\mathrm{BAV}$ as reference, figure 3B). There was a $44.4 \%$ higher risk of AVR after repeat SAV compared with BAV, but this was not statistically significant. The subgroup sensitivity analysis showed that this effect was confounded by the subgroup with associated cardiac defects. After undergoing a reintervention AVR, the probability of a second AVR was significantly lower (HR $0.3, \mathrm{p}=0.04$ ).

\section{Freedom from BAV/SAV reinterventions}

The unadjusted freedom from BAV reintervention at 10 years was $82.2 \%(76.5 ; 86.7 \%)$ after initial $\mathrm{BAV}, 73.8 \%$ $(62.3 ; 82.2 \%)$ after initial SAV and $80.2 \%(75.2 ; 84.3 \%)$ overall. Transcatheter reinterventions were significantly less frequent after a SAV or AVR reintervention (adjusted $\mathrm{HR}=0.2, \mathrm{p}=0.04$ and $\mathrm{HR}=0.05, \mathrm{p}=0.004$ respectively, initial $\mathrm{BAV}$ as reference, figure $3 \mathrm{C}$ ).

The unadjusted freedom from SAV reintervention at 10 years was $91 \%(87.3 ; 93.6 \%)$ after initial BAV, $86.6 \%$ $(74 ; 93.3 \%)$ after initial SAV and $89.6 \%$ (85.3;92.7\%) overall. Surgical reinterventions are significantly less frequent after an AVR (adjusted $\mathrm{HR}=0.1, \mathrm{p}=0.03$, initial $\mathrm{BAV}$ as reference, figure $3 \mathrm{D}$ ).

Freedom from any aortic reintervention (including AVR) at 10 years was $61.8 \%(54.9 ; 68 \%)$ after initial BAV, $55.2 \%(43.4 ; 65.4 \%)$ after initial SAV and $60.2 \%$ $(54.3 \% ; 65.7 \%)$ overall.

\section{Predictors of late outcomes}

Predictors of late survival and freedom from reintervention are summarised in table 2 .

\section{Outcomes in neonates}

Out of the 647 patients, $292(45 \%)$ were neonates at the time of the first procedure (211 in the BAV group, 81 in the SAV group). We found higher mortality in the neonatal group compared with the infant group $(10.3 \%$ vs $1.9 \%, \mathrm{p}<0.001)$, higher likelihood of AVR $(3.2 \%$ vs $0.3 \%$, $\mathrm{p}=0.006)$ and also of non-AVR reintervention $(9.2 \%$ vs $1.9 \%, \mathrm{p}<0.001)$ at 30 days. This population accounted for 29/35 (82\%) of deaths, 9/10 (90\%) of AVRs and 26/32 $(81 \%)$ of non-AVR reinterventions occurring early after the initial procedures, without significant differences due to choice between BAV and SAV. All late outcomes were worse in the neonatal subgroup (table 2).

\section{Outcomes in patients with associated cardiac defects}

The proportion of patients with associated cardiac defects was significantly higher in the SAV group when compared with the BAV group (50.2\% vs $23.2 \%$, $\mathrm{p}<0.001$ ) as was the proportion of those undergoing concomitant procedures $(22 \%$ vs $2 \%, \mathrm{p}<0.001)$. The only type of concomitant procedures observed in the BAV group was treatment of CoA.

In total, there were 193 patients with associated cardiac defects (113 with BAV, 80 with SAV). Out of these, 45 $(23.3 \%)$ underwent concomitant procedures at the initial repair (10 with BAV and 35 with SAV). They had lower survival ( $86.9 \%$ vs $92.7 \%, \mathrm{p}=0.04)$, similar freedom from AVR and lower freedom from any aortic valve reintervention $(52.3 \%$ vs $63.6 \%, \mathrm{p}<0.001)$ at 10 years when compared with those with isolated aortic valve disease. In most cases undergoing initial SAV, there were concomitant repairs of other defects $(35 / 80,26$ of which were CoA repair), with fewer concomitant transcatheter procedures in the BAV group (10/113, all CoA dilations). SAV with concomitant repairs was associated with a trend towards increased late mortality compared with isolated $\mathrm{SAV}$, although not statistically significant (HR 2.3, CI 0.8 to $6.1, \mathrm{p}=0.09$ ).

Patients with concomitant cardiac defects in the BAV and SAV groups were also matched based on the age, weight and associated defect types. This resulted in 54 patients with initial BAV and 26 with an initial SAV. An association between the choice for initial SAV and increased mortality was found, although not statistically significant, in both unmatched patients with associated cardiac defects (HR 2.3, CI 0.9 to 5.4, $\mathrm{p}=0.07$, initial BAV as reference) and matched groups (HR 2.9, CI 0.9 to 9.3, 

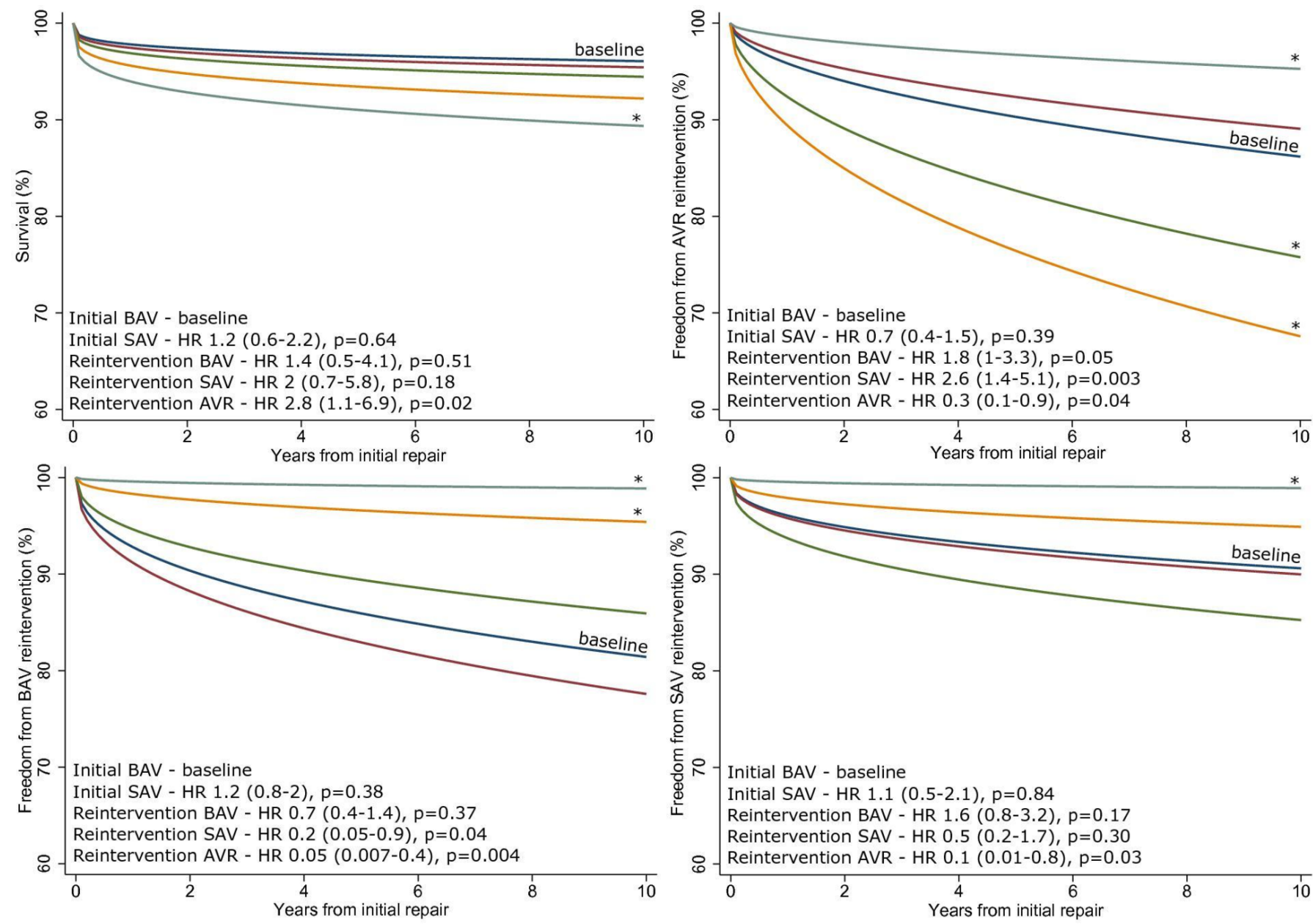

\begin{tabular}{lcccccc} 
& \multicolumn{7}{c}{ Patients at risk } \\
Years from initial repair & 0 & 2 & 4 & 6 & 8 & 10 \\
\hline Initial BAV & 488 & 317 & 240 & 189 & 137 & 98 \\
Initial SAV & 159 & 81 & 59 & 48 & 42 & 35 \\
Reintervention BAV & 98 & 57 & 35 & 26 & 20 & 13 \\
Reintervention SAV & 59 & 30 & 22 & 15 & 9 & 5 \\
Reintervention AVR & 84 & 68 & 45 & 36 & 24 & 14
\end{tabular}

Figure 3 Survival and freedom from reintervention curves, adjusted by procedure type (initial/reintervention), patient demographic data, coexisting defects (variables shown in table 2). Data are for mean variable values, obtained from multivariable Weibull regression, stratified by procedure type. (A) Survival, (B) freedom from AVR, (C) freedom from BAV type reintervention, (D) freedom from SAV type reintervention. * denotes statistically significant difference from the baseline (blue line). AVR, aortic valve replacement; BAV, balloon aortic valvoplasty; SAV, surgical aortic valvotomy.

$\mathrm{p}=0.07$, initial BAV as reference) and possibly confounded by concomitant surgical procedures. The small number of patients undergoing isolated initial SAV did not lend itself to a separate analysis.

\section{DISCUSSION}

To our knowledge, this is the largest series of neonates and infants treated with either BAV or SAV and it describes all consecutive patients from a large multicentre national registry. At 10 years after the initial $\mathrm{BAV}$ or $\mathrm{SAV}$, we report $89.3 \%$ survival and $78.7 \%$ freedom from AVR. We found no significant differences in outcomes at 10 years between BAV and SAV, after adjusting for covariates, including age and reinterventions. When BAV or SAV are performed as a reintervention, they are both associated with similarly higher rates of AVR. Neonatal intervention is associated with worse outcomes than in infants, without differences due to BAV or SAV choice. In the subgroup of patients with coexisting cardiac defects, we found a trend for higher mortality at 10 years associated with initial SAV and performing other concomitant cardiac procedures, although both comparisons were underpowered.

The number of patients with AS undergoing treatment, be it surgical or transcatheter has been steadily increasing, as evidenced by the data in figure 1 . The choice between BAV and SAV in very young patients is a matter of ongoing debate and has been so for decades. ${ }^{2}$ This is evidenced by how the ratio between the two approaches has changed through the last decade in the UK, with SAV use gradually declining in the favour of BAV in the years following the landmark study by McCrindle et al in 2001. ${ }^{4}$ Since the practice is not uniform, there were centres still 
Table 2 Predictors of mortality and reinterventions after $\mathrm{BAV}$ and SAV procedures

\begin{tabular}{|c|c|c|c|}
\hline & HR & $95 \% \mathrm{Cl}$ & $P$ value \\
\hline \multicolumn{4}{|l|}{ Mortality } \\
\hline Reintervention AVR & 2.8 & 1.1 to 6.9 & 0.02 \\
\hline $\begin{array}{l}\text { Age at current procedure } \\
\text { (years) }\end{array}$ & $0.17 /$ year & 0.03 to 0.86 & 0.03 \\
\hline $\begin{array}{l}\text { Neonate at initial } \\
\text { procedure }\end{array}$ & 3 & 1.5 to 6.2 & 0.003 \\
\hline $\begin{array}{l}\text { Weight-for-age Z score at } \\
\text { current procedure }\end{array}$ & 0.78/unit & 0.67 to 0.91 & 0.02 \\
\hline SAS & 0.1 & 0.02 to 1 & 0.05 \\
\hline VSD & 3.6 & 1.7 to 7.8 & 0.001 \\
\hline \multicolumn{4}{|l|}{ AVR } \\
\hline Reintervention $\mathrm{BAV}^{*}$ & 1.9 & 1 to 3.4 & 0.04 \\
\hline Reintervention SAV* & 2.6 & 1.4 to 5.1 & 0.004 \\
\hline Reintervention AVR* & 0.3 & 0.1 to 0.9 & 0.04 \\
\hline $\begin{array}{l}\text { Neonate at initial } \\
\text { procedure }\end{array}$ & 2.1 & 1.3 to 3.5 & 0.002 \\
\hline $\begin{array}{l}\text { Weight-for-age } Z \text { score at } \\
\text { current procedure }\end{array}$ & 0.84/unit & 0.74 to 0.96 & 0.01 \\
\hline SAS & 2.3 & 1.3 to 4.3 & 0.005 \\
\hline Female gender & 0.6 & 0.3 to 1 & 0.05 \\
\hline \multicolumn{4}{|l|}{ BAV } \\
\hline Reintervention SAV* & 0.2 & 0.05 to 0.9 & 0.04 \\
\hline Reintervention AVR ${ }^{\star}$ & 0.05 & 0.007 to 0.4 & 0.004 \\
\hline $\begin{array}{l}\text { Neonate at initial } \\
\text { procedure }\end{array}$ & 2.4 & 1.5 to 3.6 & $<0.001$ \\
\hline SAS & 2.8 & 1.6 to 4.9 & $<0.001$ \\
\hline \multicolumn{4}{|l|}{ SAV } \\
\hline Reintervention AVR* & 0.1 & 0.01 to 0.8 & 0.03 \\
\hline $\begin{array}{l}\text { Neonate at initial } \\
\text { procedure }\end{array}$ & 2.4 & 1.4 to 4.2 & 0.002 \\
\hline MV defect & 3.1 & 1.7 to 5.9 & $<0.001$ \\
\hline
\end{tabular}

${ }^{*}$ Reference is initial BAV.

AVR, aortic valve replacement; BAV, balloon aortic valvoplasty; $\mathrm{MV}$, mitral valve; SAS, subaortic stenosis; SAV, surgical aortic valvotomy; VSD, ventricular septal defect.

opting for the surgical approach, both in the UK and outside, with good results, as shown by the multiple series promoting SAV which were published after $2010 .{ }^{56}$ The upward trend of SAV use seen in the late decade is very likely a result of centres looking into their own practices and good surgical results, as no important SAV series preceded these changes. Having very little high quality evidence led to fluid practices, influenced in equal terms by published work and individual centre results.

Our data on outcomes for aortic valve stenosis treatment are comparable to those reported by Hill et al in a recent meta-analysis including 483 neonates and infants, ${ }^{3}$ with the exception that we did not find higher reintervention rates after BAV. This is likely due to the fact that in our study it was possible to adjust for multiple factors which were adding selection bias in other studies, an issue highlighted by the authors of the meta-analysis. In the first multicentre study comparing the two options in neonates, McCrindle and colleagues also do not report differences in reintervention rates. ${ }^{4}$

This report sheds light on outcomes of a large group of children who required aortic valve (non-AVR) reintervention after an initial BAV or SAV, showing that they have a higher rate of later AVR during childhood, especially when they have associated cardiac defects. Crespo et al have previously shown a less effective peak gradient reduction in reintervention $\mathrm{BAV}$, but do not report hard outcomes such as mortality or reintervention. ${ }^{10}$ Since balloon valvoplasty is traditionally considered to be the more damaging to the cusp structure out of the two options, ${ }^{11}$ it is reassuring that in fact when repeat procedures are done, reintervention BAV and SAV appear comparable in terms of survival.

In the subgroup analysis on neonates, the results are less good. This subgroup accounted for almost half of total patients, but close to $90 \%$ of all early deaths or reinterventions observed overall. The outcomes, both early and late, were similar regardless of choice between BAV and SAV, as was found in the multicentre study by McCrindle $e t$ al and the meta-analysis by Hill $e t a l .^{34}$

Another issue is the strategy for patients with associated cardiac defects. Traditionally if more than one lesion can be surgically addressed a larger procedure is planned and multiple defects are corrected. While this is based on sound clinical reasoning to avoid multiple operations, it does invite the question of how to proceed when one or more of the surgical steps can be performed percutaneously instead. To look into this issue, we studied the subgroup with associated cardiac defects. As expected, most of those undergoing SAV had multiple corrections performed at the index procedure (mostly CoA repair), while the only concomitant procedure performed in the BAV group was CoA dilation. Interestingly, we found that those with initial SAV, and among them those with concomitant repairs at index, had a higher late mortality, although not statistically significant. Even based on this underpowered comparison, it is reasonable to say that the hypothesis of better outcomes with staged, hybrid repair of complex cardiac defects would warrant testing in a prospective study, with better control of indication bias.

Finding no significant differences between BAV and SAV in terms of mortality, AVR and reintervention rates is encouraging, as it permits for a scenario-based decision rather than one imposed by perceived differences in outcomes. Our data support the consensus that both methods should be available as choices, as each patient will likely benefit more from one or the other initially and even both throughout their lifetime. ${ }^{11-13}$ New imaging techniques, especially those offering high resolution 3D reconstructions, could offer more future insight into factors influencing choice. 


\section{Limitations}

This study is limited by its retrospective nature and lack of clinical details such as echocardiographic, valve structure or reasoning for choosing a treatment option data. The large, multicentre setting and full procedural coverage partly reduces bias by allowing for multivariable adjustment and including patients from centres favouring either method, but there are still confounders which cannot be accounted for, including centre preference. The lack of functional data did not permit for endpoints such as ventricular function, transvalvular gradient or regurgitation severity to be assessed. The data request processing delay and administrative changes did not allow for the most recent 5 years to be included in the manuscript. Not having updated life status for $7.7 \%$ of patients might decrease the accuracy of statistical comparisons, but due to the random, administrative nature of the missing data, the risk of clinical bias is minimal.

\section{CONCLUSION}

In a large retrospective multicentre national registry of neonates and infants with critical aortic valve stenosis, both transcatheter valvoplasty and surgical valvotomy achieve comparable survival and freedom from reintervention at 10 years in unselected patients. Intervention choice can and should be individualised for each case, based on operative risk, valve anatomy and future treatment plans and not on perceived outcomes alone. When neonatal intervention or aortic valve reinterventions are needed, outcomes are significantly worse, a likely reflection of more dysplastic valves and precarious patient condition. The management strategy of patients with aortic valve stenosis and concomitant cardiac defects should be re-evaluated in prospective studies, as a staged approach including a transcatheter aortic valvoplasty appears to be associated with a lower mortality.

Acknowledgements This research was supported by National Institute for Health Research (NIHR) Bristol Cardiovascular Biomedical Research Centre and used data provided by the National Institute for Cardiovascular Outcomes Research, as part of the National Congenital Heart Disease Audit (NCHDA). The NCHDA is commissioned by the Healthcare Quality Improvement Partnership (HQIP) as part of the National Clinical Audit and Patient Outcomes Programme (NCAPOP) and within the National Health Service, UK. The views expressed are those of the authors and not necessarily those of the National Health Service, NIHR or Department of Health.

Contributors DMD, RMRT, DT and SCS contributed to the conception or design of the work. DMD, MTAS and EMA on data collection, analysis and interpretation. DMD, RMRT, DT, GDV and SCS on drafting the article. RMRT, DT and GDV on critical revision of the article. All coauthors gave final approval for this version to be published.

Funding This research was supported by National Institute for Health Research (NIHR) Bristol Cardiovascular Biomedical Research Centre.

Competing interests None declared.

Patient consent for publication Not required.

Ethics approval This study complies with the Declaration of Helsinki and received ethics approval from the NICOR Research Board. Patient level consent for this retrospective study using anonymised data was waived by the NICOR Research Board.

Provenance and peer review Not commissioned; externally peer reviewed.

Data sharing statement № additional data are available.

Open access This is an open access article distributed in accordance with the Creative Commons Attribution Non Commercial (CC BY-NC 4.0) license, which permits others to distribute, remix, adapt, build upon this work non-commercially, and license their derivative works on different terms, provided the original work is properly cited, appropriate credit is given, any changes made indicated, and the use is non-commercial. See: http://creativecommons.org/licenses/by-nc/4.0/.

\section{REFERENCES}

1. Kitchiner D, Jackson M, Malaiya N, et al. Incidence and prognosis of obstruction of the left ventricular outflow tract in Liverpool (1960-91): a study of 313 patients. Heart 1994;71:588-95.

2. Backer CL. Infant congenital aortic valve stenosis: the pendulum swings. J Am Coll Cardiol 2013;62:2141-3.

3. Hill GD, Ginde S, Rios R, et al. Surgical valvotomy versus balloon valvuloplasty for congenital aortic valve stenosis: a systematic review and meta-analysis. J Am Heart Assoc 2016;5:e003931.

4. McCrindle BW, Blackstone EH, Williams WG, et al. Are outcomes of surgical versus transcatheter balloon valvotomy equivalent in neonatal critical aortic stenosis? Circulation 2001;104-152-8.

5. Brown JW, Rodefeld MD, Ruzmetov M, et al. Surgical valvuloplasty versus balloon aortic dilation for congenital aortic stenosis: are evidence-based outcomes relevant? Ann Thorac Surg 2012;94:146-55.

6. Siddiqui J, Brizard CP, Galati JC, et al. Surgical valvotomy and repair for neonatal and infant congenital aortic stenosis achieves better results than interventional catheterization. J Am Coll Cardiol 2013;62:2134-40.

7. Hochstrasser L, Ruchat P, Sekarski N, et al. Long-term outcome of congenital aortic valve stenosis: predictors of reintervention. Cardiol Young 2015;25:893-902.

8. Blackwell M, lacus S, King G, et al. CEM: Coarsened exact matching in Stata. The Stata Journal 2009;9:524-46.

9. Dorobantu DM, Pandey R, Sharabiani MT, et al. Indications and results of systemic to pulmonary shunts: results from a national database. Eur J Cardiothorac Surg 2016;49:1553-63.

10. Crespo D, Miró J, Vobecky SJ, et al. Experience in a single centre with percutaneous aortic valvoplasty in children, including those with associated cardiovascular lesions. Cardiol Young 2009;19:372-82.

11. d'Udekem $Y$, Tweddell JS, Karl TR. The great debate series: surgical treatment of aortic valve abnormalities in children. Eur J CardioThoracic Surg 2018;53:919-31.

12. Hraška V. Neonatal aortic stenosis is a surgical disease. Semin Thorac Cardiovasc Surg Pediatr Card Surg Annu 2016;19:2-5.

13. Benson L. Neonatal aortic stenosis is a surgical disease: an interventional cardiologist view. Semin Thorac Cardiovasc Surg Pediatr Card Surg Annu 2016;19:6-9. 